\title{
Gamma Strength Functions and Level Densities from High-Resolution Proton Scattering under $0^{\circ}$
}

\author{
Peter von Neumann-Cosel ${ }^{1}$, a , Sergej Bassauer ${ }^{1}$, Dirk Martin ${ }^{1}$, and Atsushi Tamii ${ }^{2}$ \\ ${ }^{1}$ Institut für Kernphysik, Technische Universität Darmstadt, 64289 Darmstadt, Germany \\ ${ }^{2}$ Research Center for Nuclear Physics, Osaka University, Ibaraki, Osaka 567-0047, Japan
}

\begin{abstract}
Inelastic proton scattering at energies of a few $100 \mathrm{MeV}$ and forward angles including $0^{\circ}$ provides a novel method to measure gamma strength functions (GSF) in nuclei in an energy range of about $5-20 \mathrm{MeV}$. The experiments provide not only the E1 but also the M1 part of the GSF. The latter is poorly known in heavy nuclei. Comparison with gamma decay data (e.g. from the Oslo method) allows to test the generalised BrinkAxel (BA) hypothesis in the energy region of the pygmy dipole resonance (PDR) crucial for the modelling of $(\mathrm{n}, \gamma)$ and $(\gamma, \mathrm{n})$ reactions in astrophysical reaction networks. From the two test cases studied, ${ }^{208} \mathrm{~Pb}$ remains inconclusive in the energy region of the PDR because of large Porter-Thomas fluctuations due to the small level density (LD), while the BA hypothesis seems to hold in case of ${ }^{96} \mathrm{Mo}$. A fluctuation analysis of the high-resolution data also provides a direct measure of the LD in the energy region of the isovector giant dipole resonance (IVGDR) well above the neutron threshold, where hardly any experimental information is available. This permits an independent test of the decomposition of GSF and LD in Oslo-type experiments.
\end{abstract}

\section{Introduction}

The gamma strength function describes the average $\gamma$ decay behavior of a nucleus. Their knowledge is required for applications of statistical nuclear theory in astrophysics [1], reactor design [2], and waste transmutation [3]. The GSF is dominated by E1 radiation with smaller contributions from M1 strength, while higher multipoles contribute little. Above particle threshold it is governed by the IVGDR but at lower excitation energies one observes In nuclei with neutron excess the formation of a PDR [4] sitting on the low-energy tail of the IVGDR. This low-energy E1 strength impacts on astrophysical reaction rates and the resulting abundances in the $r$ process [5-7].

Most applications imply an environment of finite temperature, notably in stellar scenarios [8], and thus reactions on initially excited states become relevant. Their contributions to the reaction rates are usually estimated applying the generalized Brink-Axel (BA) hypothesis $[9,10]$, which states that the GSF is independent of the properties of the initial and final states and thus should be the same in $\gamma$ emission and absorption experiments. Although historically formulated for the IVGDR, where it seems to hold approximately for not too high temperatures [11], this is nowadays a commonly used assumption to calculate the low-energy E1 and M1 strength functions. Recent theoretical studies $[12,13]$ put that into question demonstrating that the strength functions of collective modes built on excited states do show an energy dependence. However, numerical results for E1 strength functions obtained

\footnotetext{
ae-mail: vnc@ikp.tu-darmstadt.de
}

in Ref. [12] showed an approximate constancy as a function of excitation energy consistent with the BA hypothesis.

Recent work utilizing compound nucleus $\gamma$ decay with the so-called Oslo method [14] has demonstrated independence of the GSF from excitation energies and spins of initial and final states in a given nucleus in accordance with the BA hypothesis once the LD is sufficiently large to suppress strong intensity fluctuations of individual transitions [15]. However, there are a number of experimental results which indicate violations of the BA hypothesis in the low-energy region. For example, the GSF in heavy deformed nuclei at excitation energies of $2-3 \mathrm{MeV}$ is dominated by the orbital M1 scissors mode [16] and potentially large differences in $B(\mathrm{M} 1)$ strengths are observed between $\gamma$ emission and absorption experiments [17-19]. At very low energies $(<2 \mathrm{MeV})$ an increase of GSFs is observed in Oslo-type experiments [20, 21], which for even-even nuclei cannot have a counterpart in ground state absorption experiments because of the pairing gap.

For the low-energy E1 strength in the region of the $\mathrm{PDR}$, the question is far from clear when comparing results from the Oslo method with photoabsorption data. Below particle thresholds most information on the GSF stems from nuclear resonance fluorescence (NRF) experiments. A striking example of disagreement is the GSF of ${ }^{96} \mathrm{Mo}$, where the results from the Oslo method $[22,23]$ and from NRF [24] differ by factors $2-3$ at excitation energies between 4 and $7 \mathrm{MeV}$. A problem with the NRF method are the experimentally unobserved braching ratios to excited states. While many older analyses of NRF data assume 
that these are negligible, in Ref. [24] they are included by a Hauser-Feshbach calculation assuming statistical decay. The resulting corrections are sizable, reaching a factor of five close to the neutron threshold. On the other hand, there are indications of non-statistical decay behavior of the PDR from recent measurements [25, 26]. Violation of the BA hypothesis was also claimed in a simultaneous study of the $\left(\gamma, \gamma^{\prime}\right)$ reaction and average ground state branching ratios [27] in ${ }^{142} \mathrm{Nd}$ (see, however, Ref. [28]). Clearly, information on the GSF - in particular in the energy region of the PDR - from independent experiments is of high interest.

A new method for the measurement of complete E1 strength distributions - and thereby the E1 part of the GSFs - in nuclei from about 5 to $20 \mathrm{MeV}$ has been developed using relativistic Coulomb excitation in polarized inelastic proton scattering at energies of a few hundred $\mathrm{MeV}$ and scattering angles close to $0^{\circ}$ [29-33]. These data allow the dipole polarizability to be determined which provides important constraints on the neutron skin of nuclei and the poorly known parameters of the symmetry energy [34]. They also permit extraction of the M1 part of the GSF due to spinflip excitations [35], which energetically overlaps with the PDR strength. Furthermore, when performed with good energy resolution, the level density can be extracted in the excitation region of the IVGDR from the giant resonance fine structure independent of the GSF [36]. This allows an important test of the model-dependent decomposition of LD and GSF in the Oslo method [14] .

\section{Experiments and and multipole decomposition}

By way of example we explain the the experimental methods and major steps of the data analysis with the data for ${ }^{96}$ Mo [37]. Further details can be found in Ref. [38]. Figure 1(a) displays the spectra taken at spectrometer angles $0^{\circ}, 3^{\circ}$, and $4.5^{\circ}$. Besides discrete transitions at low excitation energies, a resonance-like structure around $8 \mathrm{MeV}$ and the prominent IVGDR centered at $E_{x} \approx 16 \mathrm{MeV}$ are observed. The cross sections show a strong forward-angle peaking indicating the dominance of Coulomb excitation. The total spin transfer

$$
\Sigma=\frac{3-\left(2 D_{N N^{\prime}}+D_{L L^{\prime}}\right)}{4}
$$

shown in Fig. 1(b) can be extracted from the measured polarization transfer observables. It takes a value of one for spinflip $(\Delta S=1)$ and zero for non-spinflip $(\Delta S=0)$ transitions at $0^{\circ}$ [39], interpreted as M1 and E1 cross sections parts, respectively. Values in between 0 and 1 result from the summation over finite energy bins ( $200 \mathrm{keV}$ up to an excitation energy of $10 \mathrm{MeV}$ and $500 \mathrm{keV}$ above). The polarization transfer analysis (PTA) reveals the presence of a few spinflip transitions between 5 and $7.5 \mathrm{MeV}$ and a concentration of spinflip strength in the energy region 7.5-10 $\mathrm{MeV}$ identified as the spin-M1 resonance in ${ }^{96} \mathrm{Mo}$. Cross sections above $10 \mathrm{MeV}$ are dominantly of $\Delta S=0$ character as expected for Coulomb excitation. These findings
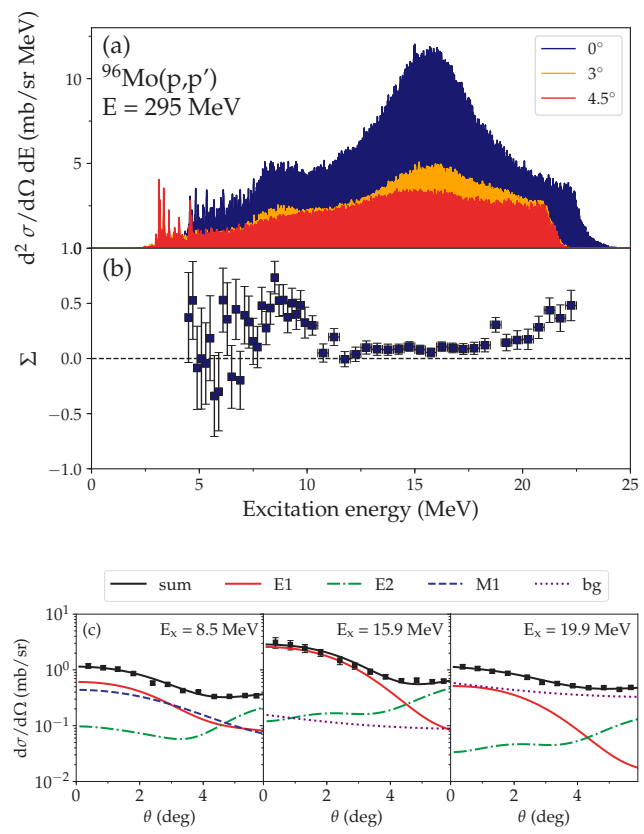

Figure 1. (a) Spectra of the ${ }^{96} \mathrm{Mo}\left(\overrightarrow{\mathrm{p}}, \overrightarrow{\mathrm{p}}^{\prime}\right)$ reaction at $E_{p}=295$ $\mathrm{MeV}$ with the spectrometer placed at $0^{\circ}$ (blue), $3^{\circ}$ (yellow), and $4.5^{\circ}$ (red). (b) Total spin transfer $\Sigma$, Eq. (1). (c) Examples of the MDA for selected energy bins. From Ref. [37].

are consistent with the results in ${ }^{208} \mathrm{~Pb}$ [29] and ${ }^{120} \mathrm{Sn}$ [32]. The $\Delta S=1$ strength observed at high $E_{\mathrm{x}}$ can be understood to arise from quasi-free scattering [40].

Alternatively, a multipole decomposition analysis (MDA) was performed for angular distributions of the cross sections in the PDR and GDR regions. For this purpose, angular cuts were applied to the spectra of Fig. 1(a) providing 4 data points each. The MDA followed closely the approach described in Refs. [29, 30]. For the nuclear background the empirical parametrization found for ${ }^{208} \mathrm{~Pb}$ [36] was adopted assuming the same momentum transfer dependence. Figure 1(c) presents representative examples of the MDA for $200 \mathrm{keV}$ excitation energy bins at different excitation energies. They demonstrate that in the lowenergy bump M1 contributions are sizable while E1 dominates in the energy region of the IVGDR. At even higher energies, the nuclear background becomes dominant.

\section{Gamma strength functions of ${ }^{96} \mathrm{Mo}$ and ${ }^{208} \mathrm{~Pb}$}

After the decomposition of E1 and M1 cross sections described above, both can be converted into photoabsorption cross sections, or equivalently, the GSF. For the E1 strength this is based on the virtual photon method of relativistic Coulomb excitation [41]. Spin M1 matrix elements can be derived from the M1 cross sections utilizing 
the unit cross section method and further converted into equivalent electromagnetic B(M1) transition strengths neglecting isoscalar and orbital contributions [35]. The former assumption is motivated by the strong suppression of isoscalar strength due to the anomalous nucleon magnetic moments, while the latter is well justified in the energy region of the spin M1 resonanance [17].

Figure 2 presents the E1 (blue), M1 (green), and E2 (red) GSF in ${ }^{208} \mathrm{~Pb}$ [42]. The latter is deduced from the isoscalar E2 strength function given in Ref. [43]. As can be seen, the dominant contribution stems from E1 transitions. The M1 contribution is of the order of a few percent for excitation energies above $8 \mathrm{MeV}$ and reaches at most $10-30 \%$ in the peak of the resonance around the neutron threshold. We note that the dominance of the E1 GSF even in the energy region of the spin M1 resonance - is a common feature in medium-heavy and heavy nuclei.

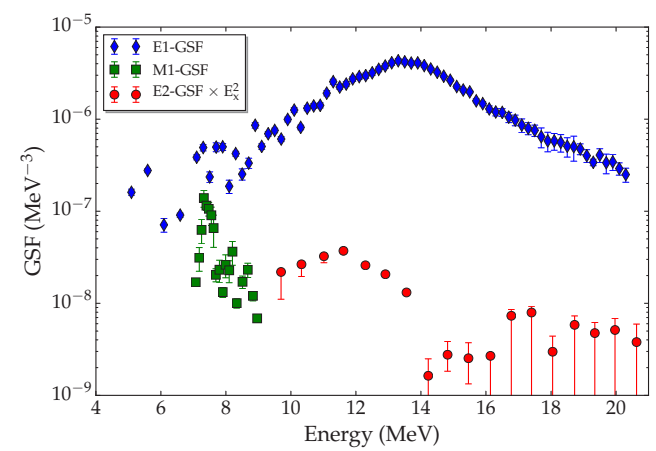

Figure 2. Comparison of E1, M1 and E2 contributions to the GSF of ${ }^{208} \mathrm{~Pb}$. From Ref. [42].

The total GSF summing all contributions is displayed in Fig. 3 (blue diamonds) and compared with data derived with the Oslo method from a ${ }^{208} \mathrm{~Pb}\left({ }^{3} \mathrm{He},{ }^{3} \mathrm{He}^{\prime} \gamma\right)$ experiment (red circles) [44]. The data set has been reanalyzed recently, for details see Ref. [42]. There are overlapping results from both experiments in the energy region between 5 and $8 \mathrm{MeV}$ (inlet of Fig. 3). The GSF derived from the $\left(\mathrm{p}, \mathrm{p}^{\prime}\right)$ data is systematically higher in the PDR region although they seem still compatible within error bars in the peak region around the neutron threshold. Between 6 and $7 \mathrm{MeV}$ consistent results are found while below $6 \mathrm{MeV}$ the strong transitions observed in Ref. [30] exceed the average $\gamma$ strength in the Oslo data by factors 4 to 5 . However, one should be aware that single transitions are analyzed for excitation energies $E_{\mathrm{x}}<7 \mathrm{MeV}$ [30] and the level density of $1^{-}$states excited from the ground state is probably too low to discuss an average behavior in the PDR region. Rather the upward GSF is dominated by Porter-Thomas intensity fluctuations preventing a conclusion on the BA hypothesis.

A better suited case to test the BA hypothesis in the energy region of the PDR is ${ }^{96} \mathrm{Mo}$, where the LD should be sufficiently high because of the considerable ground state

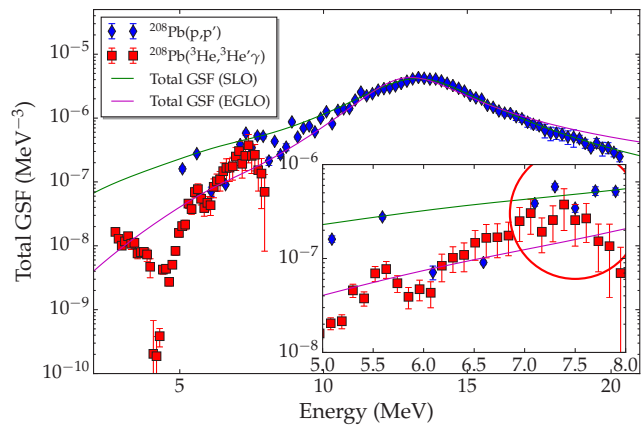

Figure 3. Total GSF of ${ }^{208} \mathrm{~Pb}$ from the $\left(\mathrm{p}, \mathrm{p}^{\prime}\right)$ data $[29,30]$ in comparison to the reanalyzed [42] results from the Oslo experiment [44]. The inlet shows an expanded view of the low-energy region 5-8 MeV. From Ref. [42].

deformation. The case of ${ }^{96} \mathrm{Mo}$ is of particular interest because of a large discrepancy of the GSF deduced from the Oslo method [22, 23] and from $\left(\gamma, \gamma^{\prime}\right)$ experiments [24]. The GSF function deduced from the $\left(\overrightarrow{\mathrm{p}}, \overrightarrow{\mathrm{p}}^{\prime}\right)$ experiment discussed above [37] is displayed in Fig. 4 together with data from other experiments. Fig. 4(a) gives an overwiev for for the full range $E_{\gamma}=6-20 \mathrm{MeV}$. The result is compared with $\left({ }^{3} \mathrm{He},{ }^{3} \mathrm{He}^{\prime} \gamma\right)[22,23]$ (open circles), $(\gamma, x n)[45]$ (grey
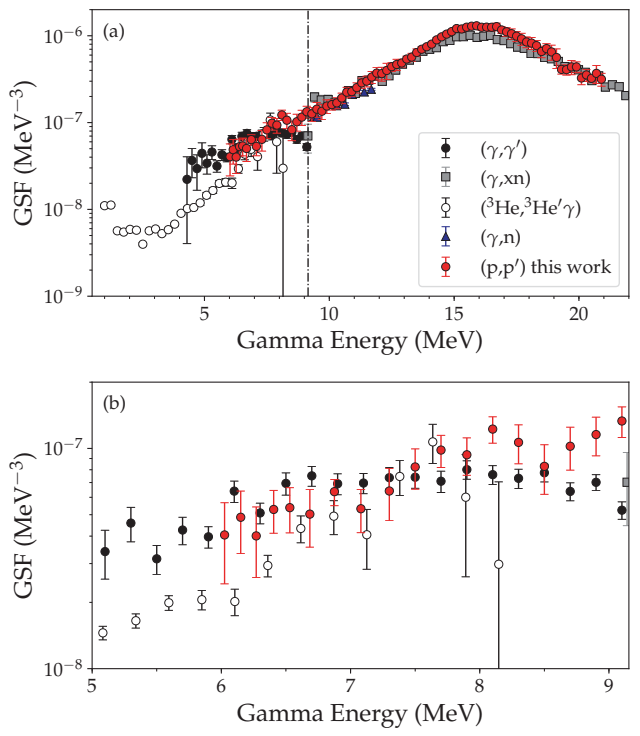

Figure 4. GSF of ${ }^{96} \mathrm{Mo}$ in the energy region from $5 \mathrm{MeV}$ to neutron threshold from the present work (red circles) compared with $\left({ }^{3} \mathrm{He}^{3}{ }^{3} \mathrm{He}^{\prime} \gamma\right)[22,23]$ (open circles), and $\left(\gamma, \gamma^{\prime}\right)[24]$ data including a statistical model correction for unobserved branching ratios (black circles). From Ref. [37]. 
squares), $(\gamma, n)[46]$ (blue upward triangles), and $\left(\gamma, \gamma^{\prime}\right)$ data corrected for unobserved branching ratios [24] (black circles).

Above threshold, there is overall fair agreement with the data from Refs. [45, 46] except that the present experiment finds somewhat larger photoabsorption cross sections around the maximum of the IVGDR. In the energy region between $5 \mathrm{MeV}$ and the neutron threshold [Fig. 4(b)] the results lie in between the two other measurements but agree within error bars with the Oslo experiment except for the lowest energies where statistics are poor.

\section{Level densities}

Since only the product of GSF and LD is measured by the Oslo method [14], it relies on external data for their decomposition. An independent check of the LD results is thus of special interest. The good energy resolution of the present data permits an extraction of the LD of $J^{\pi}=1^{-}$ states applying a fluctuation analysis to the fine structure of the IVGDR. Details of the method can be found in Refs. [36, 47, 48].

The LD of $J^{\pi}=1^{-}$states in ${ }^{208} \mathrm{~Pb}$ between 11 and $16 \mathrm{MeV}$ [36] is displayed in the inlet of Fig. 5 in comparison with different parametrizations $[44,49,50]$ of the phenomenological backshifted Fermi gas (BSFG) model. The BSFG parameters deduced from the RIPL-3 data base [49] provide a good description over the full energy range covered by the Oslo experiment and the present data. The parameterization of Ref. [50] works well at higher $E_{\mathrm{x}}$ but undershoot the data at lower $E_{\mathrm{x}}$, while the situation is reverse with the parameter set of Ref. [44]. An analog analysis of ${ }^{96} \mathrm{Mo}$ data shows similar results [37]. For the two cases studied so far, the LDs deduced from the Oslo and the present method can be consistently described.

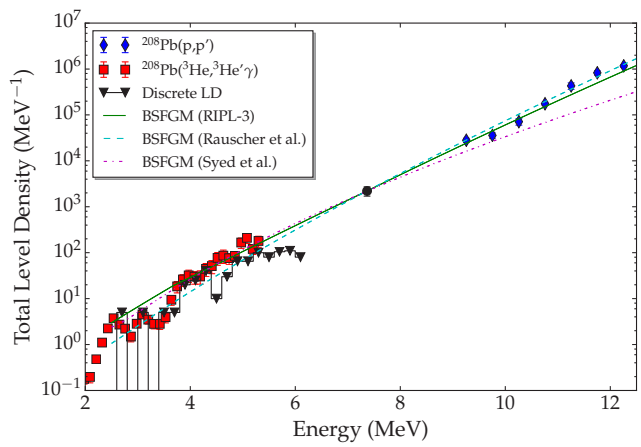

Figure 5. Total LD from the (p, $\left.\mathrm{p}^{\prime}\right)$ data $[29,30]$ in comparison to the reanalyzed results (cf. Ref. [42]) from the Oslo experiment [44]. The black downward triangles are results from counting the levels identified in Ref. [51] in $200 \mathrm{keV}$ bins. The magenta dashed-dotted, green solid, and cyan dashed lines are BSFG model predictions with the parameters of Ref. [44], Ref. [49], and Ref. [50], respectively. From Ref. [42].

\section{Concluding Remarks}

A new approach to test the Brink-Axel hypothesis is presented based on a study of the $\left(\vec{p}, \vec{p}^{\prime}\right)$ reaction at $295 \mathrm{MeV}$ and extreme forward angles. While no conclusion can be drawn for ${ }^{208} \mathrm{~Pb}$, the extracted gamma strength function ${ }^{96} \mathrm{Mo}$ agrees with results of compound nucleus $\gamma$ decay experiments $[22,23]$ indicating that the BA hypothesis holds in the energy region of the PDR, in contrast to results from the $\left(\gamma, \gamma^{\prime}\right)$ reaction [24] and the claims of Ref. [27]. This is an important finding since the BA hypothesis constitutes a basic assumption of astrophysical reaction network calculations. The high energy resolution and selectivity of the data permits an extraction of the LD at excitation energies above the neutron threshold hardly accessible by other means. A consistent description of the LD with those of the $\gamma$ decay experiments can be achieved with BSFG models providing independent confirmation of the methods used to separate GSF and LD in Oslo-type experiments.

\section{Acknowledgements}

We are grateful to all collaborators of experiments E282 and E376 for their contributions to the present work. This work was supported by the DFG under contract SFB 1245, by the JSPS, KAKENHI Grant No. JP14740154, and by the MEX,T KAKENHI Grant No. JP25105509.

\section{References}

[1] M. Arnould, S. Goriely, and K. Takahashi, Phys. Rep. 450, 97 (2007).

[2] M. B. Chadwick et al., Nucl. Data Sheets 112, 2887 (2011).

[3] M. Salvatore and G. Palmiotti, Prog. Part. Nucl. Phys. 66, 144 (2011).

[4] D. Savran, T. Aumann, and A. Zilges, Prog. Part. Nucl. Phys. 70, 210 (2013).

[5] S. Goriely, E. Khan, and M. Samyn, Nucl. Phys. A739, 331 (2004).

[6] E. Litvinova, H. P. Loens, K. Langanke, G. MartínezPinedo, T. Rauscher, P. Ring, F.-K. Thielemann, and V. Tselyaev, Nucl. Phys. A823, 26 (2009).

[7] I. Daoutidis and S. Goriely, Phys. Rev. C 86, 034328 (2012).

[8] M. Wiescher, F. Käppeler, and K. Langanke, Annu. Rev. Astron. Astrophys. 50, 165 (2012).

[9] D. M. Brink, Ph.D. thesis, Oxford University (1955).

[10] P. Axel, Phys. Rev. 126, 671 (1962).

[11] P. F. Bortignon, A. Bracco, and R. A. Broglia, Giant Resonances: Nuclear Structure at Finite Temperature (Harwood Academic, Amsterdam, 1998).

[12] C. W. Johnson, Phys. Lett. B 750, 72 (2015).

[13] N. Quang Hung, N. Dinh Dang, and L. T. Quynh Huong, Phys. Rev. Lett. 118, 022502 (2017).

[14] A. Schiller, L. Bergholt, M. Guttormsen, E. Melby, J. Rekstad, and S. Siem, Nucl. Instrum. Methods Phys. Res., Sect. A 447, 498 (2000). 
[15] M. Guttormsen, A. C. Larsen, A. Görgen, T. Renstrom, S. Siem, T. G. Tornyi, and G. M. Tveten, Phys. Rev. Lett. 116, 012502 (2016).

[16] D. Bohle, A. Richter, W. Steffen, A. E. L.Dieperink, N. Lo Iudice, F. Palumbo, and O. Scholten, Phys. Lett. B 137, 27 (1984).

[17] K. Heyde, P. von Neumann-Cosel, and A. Richter, Rev. Mod. Phys. 82, 2365 (2010).

[18] M. Guttormsen et al., Phys. Rev. Lett. 109, 162503 (2012).

[19] C. T. Angell, R. Hajima, T. Shizuma, B. Ludewigt and B. J. Quiter, Phys. Rev. Lett. 117, 142501 (2016).

[20] A. Voinov, E. Algin, U. Agvaanluvsan, T. Belgya, R. Chankova, M. Guttormsen, G. E. Mitchell, J. Rekstad, A. Schiller, and S. Siem, Phys. Rev. Lett. 93, 142504 (2004).

[21] A. C. Larsen et al., EPJ Web of Conferences 66, 07014 (2014).

[22] M. Guttormsen et al., Phys. Rev. C 71, 044307 (2005).

[23] A. C. Larsen and S. Goriely, Phys. Rev. C 82, 014318 (2010).

[24] G. Rusev et al., Phys. Rev. C 79, 061302 (2009).

[25] C. Romig et al., Phys. Lett. B 744, 369 (2015).

[26] B. Löher et al., Phys. Lett. B 756, 72 (2016).

[27] C. T. Angell, S. L. Hammond, H. J. Karwowski, J. H. Kelley, M. Krtička, E. Kwan, A. Makinaga, and G. Rusev, Phys. Rev. C 86, 051302(R) (2012).

[28] Erratum to Ref. [27], Phys. Rev. C 91, 039901(E) (2015).

[29] A. Tamii et al., Phys. Rev. Lett. 107, 062502 (2011).

[30] I. Poltoratska et al., Phys. Rev. C 85, 041304(R) (2012).

[31] A. M. Krumbholz et al., Phys. Lett. B 744, 7 (2015).

[32] T. Hashimoto et al., Phys. Rev. C 92, 031305(R) (2015).

[33] J. Birkhan et al., Phys. Rev. Lett. 118, 252501 (2017).
[34] Topical Issue on Nuclear Symmetry Energy, edited by Bao-An Li, A. Ramos, G. Verde and I. Vidaña, Eur. Phys. J. A 50(2), (2014).

[35] J. Birkhan, H. Matsubara, P. von Neumann-Cosel, N. Pietralla, V. Yu. Ponomarev, A. Richter, A. Tamii, and J. Wambach, Phys. Rev. C 93, 041302(R) (2016).

[36] I. Poltoratska, R. W. Fearick, A. M. Krumbholz, E. Litvinova, H. Matsubara, P. von Neumann-Cosel, V. Yu. Ponomarev, A. Richter, and A. Tamii, Phys. Rev. C 89, 054322 (2014).

[37] D. Martin et al., Phys. Rev. Lett. 119, 182503 (2017).

[38] A. Tamii et al., Nucl. Instrum. Meth. A 605, 3 (2009).

[39] T. Suzuki, Prog. Theor. Phys. 321, 859 (2000).

[40] F. T. Baker et al., Phys. Rep. 289, 235 (1997).

[41] C. A. Bertulani and G. Baur, Phys. Rep. 163, 299 (1988).

[42] S. Bassauer, P. von Neumann-Cosel, and A. Tamii, Phys. Rev. C 94, 054313 (2016).

[43] D. H. Youngblood, Y.-W. Lui, H. L. Clark, B. John, Y. Tokimoto, and X. Chen, Phys. Rev. C 69, 034315 (2004).

[44] N. U. H. Syed, M. Guttormsen, F. Ingebretsen, A. C. Larsen, T. Lönnroth, J. Rekstad, A. Schiller, S. Siem, and A. Voinov, Phys. Rev. C 79, 024316 (2009).

[45] H. Beil, R. Bergère, P. Carlos, A. Leprêtre, A. De Miniac, and A. Veyssiere, Nucl. Phys. A227, 427 (1974).

[46] H. Utsunomiya et al., Phys. Rev. C 88, 015805 (2013).

[47] Y. Kalmykov et al., Phys. Rev. Lett. 96, 012502 (2006).

[48] I. Usman et al., Phys. Rev. C 84, 054322 (2011).

[49] R. Capote et al., Nucl. Data Sheets 110, 3107 (2009).

[50] T. Rauscher, F.-K. Thielemann, and K.-L. Kratz, Phys. Rev. C 56, 1613 (1997).

[51] A. Heusler, R. V. Jolos, T. Faestermann, R. Hertenberger, H.-F. Wirth, and P. von Brentano, Phys. Rev. C 93, 054321 (2016). 